\title{
The Clinical View of Immediate Implant Placement: Case Series
}

\author{
Sang-Chang Lee, DDS, MSD ${ }^{1,2 *}$, Bo-Yun Choi, DDS, MSD ${ }^{3}$ \\ 'Doctoral Student, Department of Oral \& Maxillofacial Surgery, School of Dentistry, Kyungpook National \\ University, Daegu, Korea \\ ${ }^{2}$ Specialist, BYseokgye Dental Clinic, Seoul, Korea \\ ${ }^{3}$ Dentist, BYseokgye Dental Clinic, Seoul, Korea \\ *Corresponding author: Sang-Chang Lee, BYseokgye Dental Clinic, 2 Seokgye-ro, Nowon-gu, Seoul 01895, \\ Korea. \\ Tel:+82-2-912-2275. Fax : +82-2-914-2275. E-mail : 1sc7535@hanmail.net
}

\begin{abstract}
Tooth loss is common and usually a result of disease or trauma. Therefore, the use of dental implants to replace missing teeth has a long history. Immediate implant placement is a common and acceptable clinical method. Immediate implant placement was introduced as a possible means to limit bone resorption and reduce the number of surgical procedures following tooth extraction. However, immediate implant placement is a challenging surgical procedure and requires proper case selection and surgical techniques. Furthermore, there is a lot of controversy regarding the case selection criteria for immediate implant placement. If the following conditions are present: complete debridement of inflammatory tissue, presence of bone for initial stability, and soft tissue control, immediate implant placement will be useful and successful in many cases. The purpose of this report is to illustrate the usability and various applications of immediate implants in several cases.
\end{abstract}

Keywords: Bone graft, Immediate implant, Sinus lift

pISSN 2765-7833

eISSN 2765-7841

Journal of implantology and applied sciences 2021; $25(2): 64-73$

https://doi.org/10.32542/implantology.2021007

Received: December 22, 2020

Revised: February 26, 2021

Accepted: March 12, 2021

ORCID

Sang-Chang Lee

https://orcid.org/0000-0003-4471-1958

Copyright $(\odot$ 2021. The Korean Academy of Oral \& Maxillofacial Implantology

This is an Open Access article distributed under the terms of the Creative Commons Attribution Non-Commercial License (http://creativecommons. $\mathrm{org} /$ licenses/by-nc/4.0/) which permits unrestricted non-commercial use, distribution, and reproduction in any medium, provided the original work is properly cited.

\section{I . Introduction}

The coming of implants in dentistry represents a turning point in dental clinical practice. Immediate dental implant placement refers to the insertion of an implant directly after a tooth is extracted, whereas delayed implant placement occurs at a later time. The concept of placing implants immediately after tooth removal was introduced in the 1970s. ${ }^{1}$ Currently, there is a widespread acceptance of this procedure due to its high survival rate. ${ }^{2}$

According to several studies, one advantage of immediate dental implant placement is that it preserves the bony walls and prevents the collapse of alveolar bone after extraction from resorption. ${ }^{3}$ In addition, it decreases the number of surgical procedures, reduces treatment time and cost, increases patient satisfaction, placement of implants in the same position at the extraction site with better axial placement, and more esthetic results. ${ }^{3}$ 
Numerous clinical studies have reported this technique to be a predictable procedure. Long-term studies have shown that implants have high success and survival rates. ${ }^{4-10}$ Some authors suggested that immediate implant placement is contraindicated in the presence of infections such as periodontal and periapical lesions. ${ }^{11}$ However, several experimental and clinical studies have reported that immediate implant placement with periapical pathology does not have more complications and higher failure rates than those placed in a healed area. According to these studies, immediate placement of dental implants into fresh extraction sockets with periapical endodontic lesions is not contraindicated if proper clinical procedures such as antibiotic administration, cleaning of the surgical site, and alveolar debridement are followed. ${ }^{12-14}$ Block and Kent ${ }^{15}$ summarized the indications as follows (1) traumatic loss of teeth with a small amount of bone loss; (2) tooth loss due to gross decay without purulent exudates or cellulites; (3) inability to complete endodontic therapy; (4) presence of severe periodontal bone loss without purulent exudates; and (5) adequate soft tissue health to obtain primary wound closure. However, there is a lot of controversy regarding the case selection criteria. ${ }^{16}$ This study aimed to present the various applications of the immediate implant.

\section{II . Case Report}

\section{Case 1}

A 55-year-old man presented with severe pain in the left maxillary 2nd molar.

Clinical and radiological evaluation revealed severe mobility of the left maxillary 2nd molar and a cystic lesion in the crown area of the left maxillary 3rd molar (Fig. 1A). Spontaneous bleeding, purulent exudate, and periodontal abscesses were observed. He had no history of systemic disease. Under local anesthesia, a three-sided flap was elevated and the 2 nd molar \& 3 rd molars were extracted. The cystic lesion was removed and immediate implant placement with sinus elevation (lateral approach) was performed (Fig. 1B). The primary anchorage was provided by the inferior wall of the sinus. After two
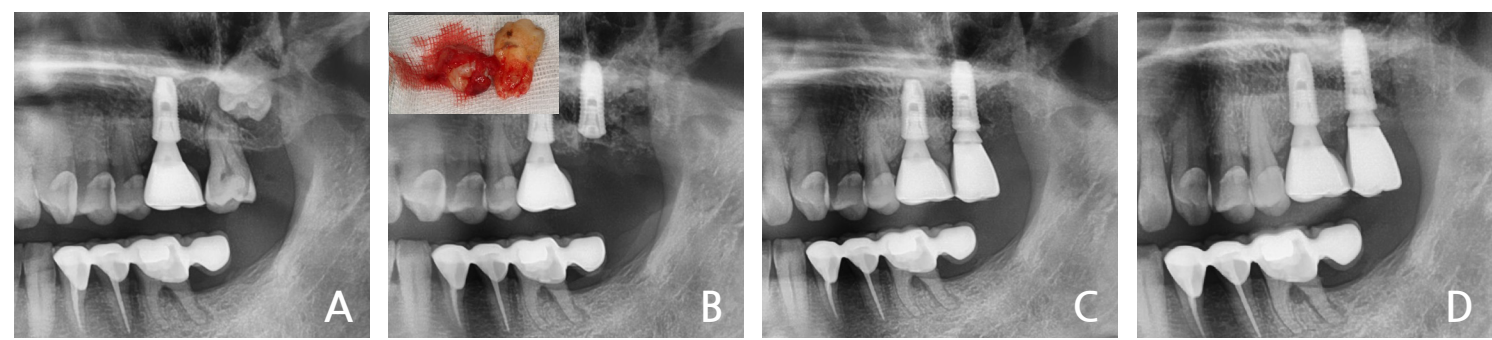

Fig. 1. (A) Cystic lesion on the coronal area of the left maxillary third molar, (B) Immediate implant placement with sinus lift after extraction of the left maxillary 2 nd $\& 3$ rd molars, (C) Loading after 2 months, (D) Panoramic view at the 3-years follow up. 
months, loading was performed (Fig. 1C). In the 3-years follow-up, the bone \& implant was intact (Fig. 1D).

\section{Case 2}

A 60-year-old man, a smoker, with no history of systemic disease visited the dental clinic complaining of severe pain in the right maxillary molars. Radiographic and clinical evaluations demonstrated severe bone loss and purulent exudates in the crevice area and bone loss in the proximal area of the right maxillary molars (Fig. 2A). Under local anesthesia, the right maxillary molars were extracted, and the infected tissue was completely removed. Immediate implant placement was performed with sinus elevation (Fig. 2B, 2C). The primary stability was attained by the inferior wall of the sinus and the remaining intact inter-septal bone. Restoration was performed after two months. After 3 years, no bone loss or preservation of the bony wall was observed (Fig. 2D).
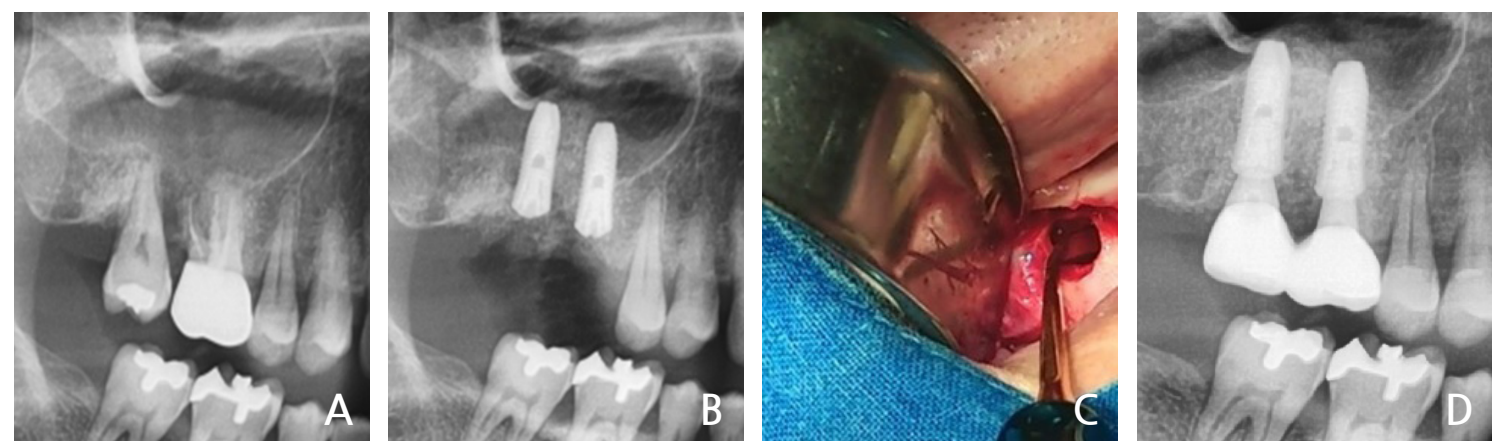

Fig. 2. (A) Bone loss at the right maxillary 1st and 2nd molars area, (B) Immediate implant placement with sinus lift, (C) Window opening, (D) Panoramic view at the 3-years follow-up.

\section{Case 3}

A 61-year old man presented to our clinic with severe pain in the right mandibular area.

He had no history of any systemic disease and was a smoker. Periapical pathology was observed on panoramic evaluation (Fig. 3A). Severe mobility was also noted. The right 1st molar was extracted under local anesthesia. After debridement, implantation with bone graft was performed at the defected bone area (Fig. 3B). The primary stability of the implant was attained by the apical 3-4 mm intact bone area. After 2 months, implant loading was performed (Fig. 3C).

After 3-years of follow-up, preservation of the gingiva and bone height was observed (Fig. 3D). 

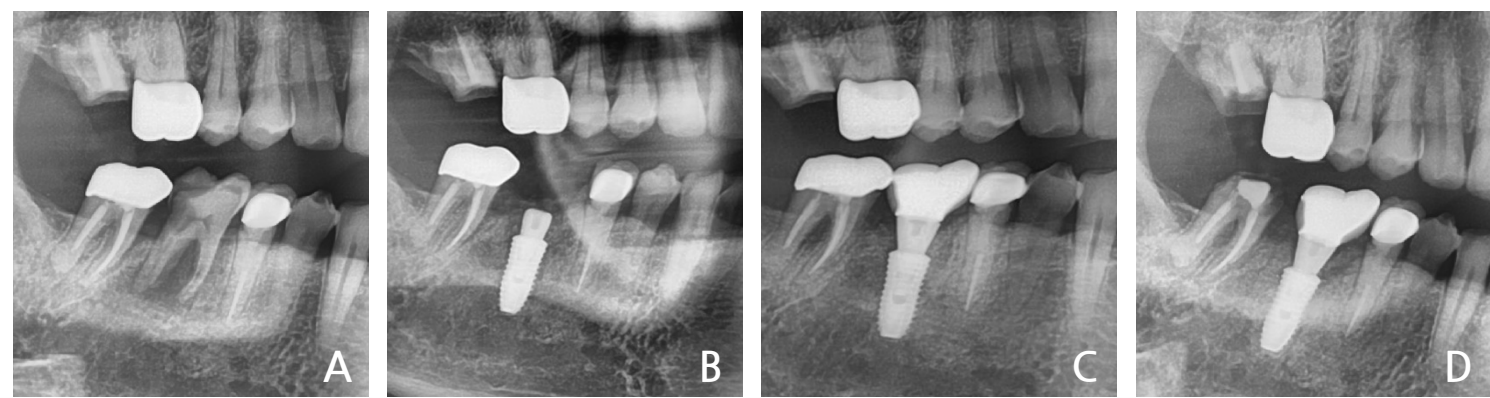

Fig. 3. (A) Periapical lesion of the right mandibular 1st molar, (B) Immediate implant placement with bone graft, (C) Loading after two months, (D) Panoramic view at the 3-years follow up.

\section{Case 4}

A 56-year-old woman presented with discomfort and pain after a bite on the right mandibular molar that had been treated at a dental clinic. She had mild-controlled hypertension. Clinical and radiographic evaluations demonstrated severe bone loss and mobility in the mandibular right 2nd premolar (Fig. 4A). Under local anesthesia, the right mandibular 2nd premolar was extracted and immediate implant placement was performed with bone graft (Fig. 4B). The primary anchorage was gained from the linguo-apical $3 \mathrm{~mm}$ intact bone. After one month, loading was performed (Fig. 4C). The radiographic evaluation after 3-years of follow-up showed that the bone level was well maintained (Fig. 4D).
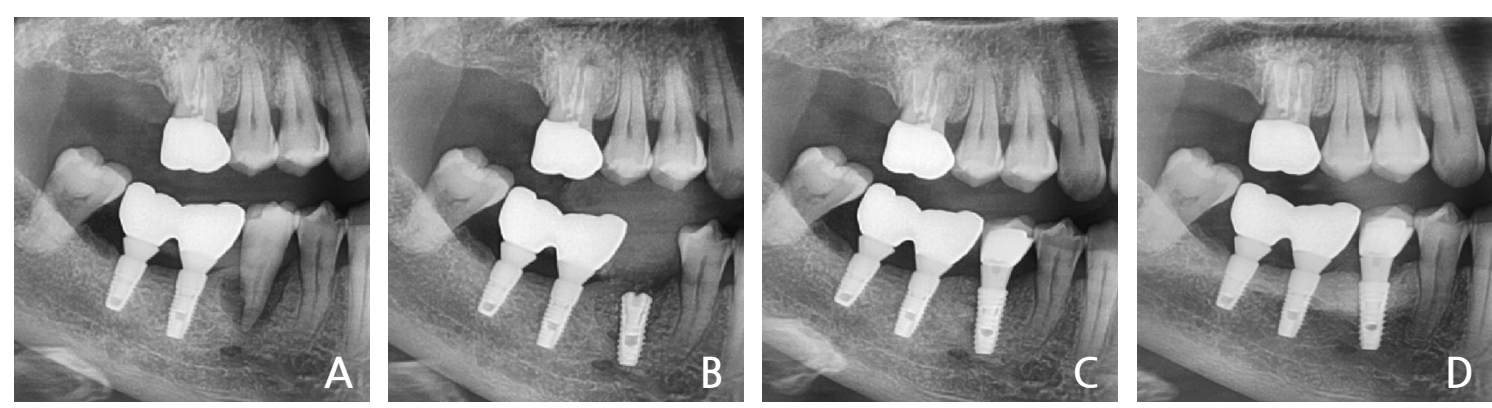

Fig. 4. (A) Periapical lesion of the mandibular 2nd premolar area, (B) Immediate implant placement with bone graft, (C) Loading after one month, (D) Panoramic view at the 3-years follow-up.

\section{Case 5}

A 68-year-old woman visited the dental clinic with the chief complaint of mobile left molar area. The patient exhibited severe mobility and purulent exudates in the left mandibular 2nd premolar - 2nd molar area. The radiographic view demonstrated a severe bone loss in the upper part of the mental foramen (Fig. 5A). The left mandibular 2nd premolar \& 2nd molar were extracted, and immediate implant placement was performed with bone graft. The primary stability of the mandibular left 2nd premolar area was achieved by the mesio-lingual $3 \mathrm{~mm}$ intact bone (Fig. 5B). After one month, the 
dental restoration was fabricated (Fig. 5C). There have been no abnormal findings in the examination for the past 3 years (Fig. 5D).
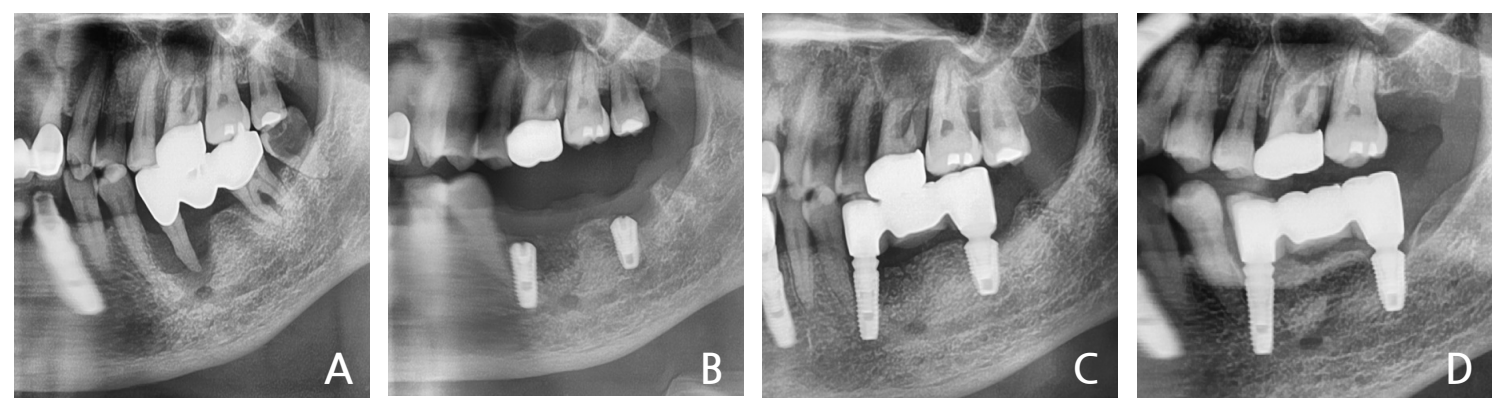

Fig. 5. (A) Severe bone resorption of the left mandibular premolar area, (B) Immediate implantation with bone graft, (C) Loading after one month, (D) Panoramic view at the 3-years follow-up.

\section{Case 6}

The patient was a 63-year-old man with no history of systemic disease. The patient visited the dental clinic complaining of bite pain of an implant placed 2 years ago at the mandibular right 2nd molar (Fig. 6A). The implant was placed by another dentist, pus discharge, severe mobility, and bone loss of the crest area were observed. The height of the residual bone was less than $6 \mathrm{~mm}$ from the intact crest to the mandibular canal. The old fixture was removed, and implantation with bone graft was performed. Primary stability was attained in the intact buccal bone area, avoiding the mandibular canal (Fig. 6B). After two months, the loading was initiated (Fig. 6C). After 2-years, the bone level was maintained in the panoramic view (Fig. 6D).
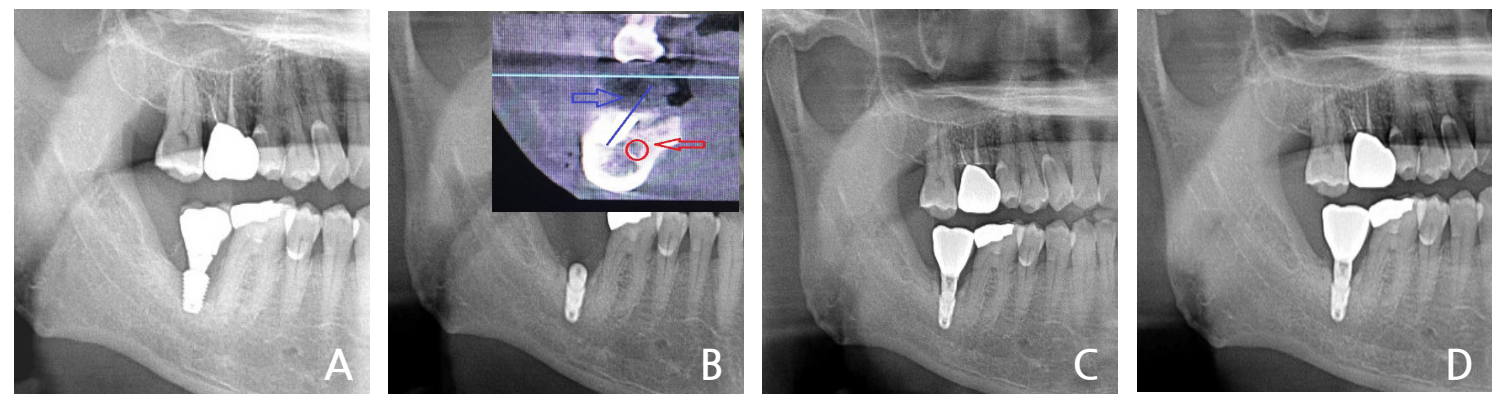

Fig. 6. (A) The bone resorption of the fixture, (B) Immediate implant placement after extraction of old fixture and fixture on the buccal area of the inferior alveolar canal (Red arrow: inferior alveolar Canal, blue arrow: fixture axis, (C) Loading after 1 month, (D) Panoramic view at the 2-years follow-up.

\section{Case 7}

A 56-year old man presenting with the chief complaint of the severe pain on the anterior maxilla visited our dental clinic. Clinical and radiographic evaluations showed severe bone loss and purulent 
exudates in the right maxillary incisor and the left molar area. The patient just wanted the treatment of the anterior maxilla area (Fig. 7A). The right maxillary incisor and left maxillary lateral incisor were therefore extracted. Immediate implant placement and bone graft were performed (Fig. 7B). The rotation flap of the distal gingiva on the right lateral incisor was performed (Fig. 7E). The primary stability was attained from the apical intact bone area. A provisional crown was made immediately after implant placement (Fig. 7E). Three weeks later, a final prosthesis was inserted. The gingival height and attached gingiva were preserved (Fig. 7G). After 3-years, the abnormal condition was not shown in the panoramic view (Fig. 7H).
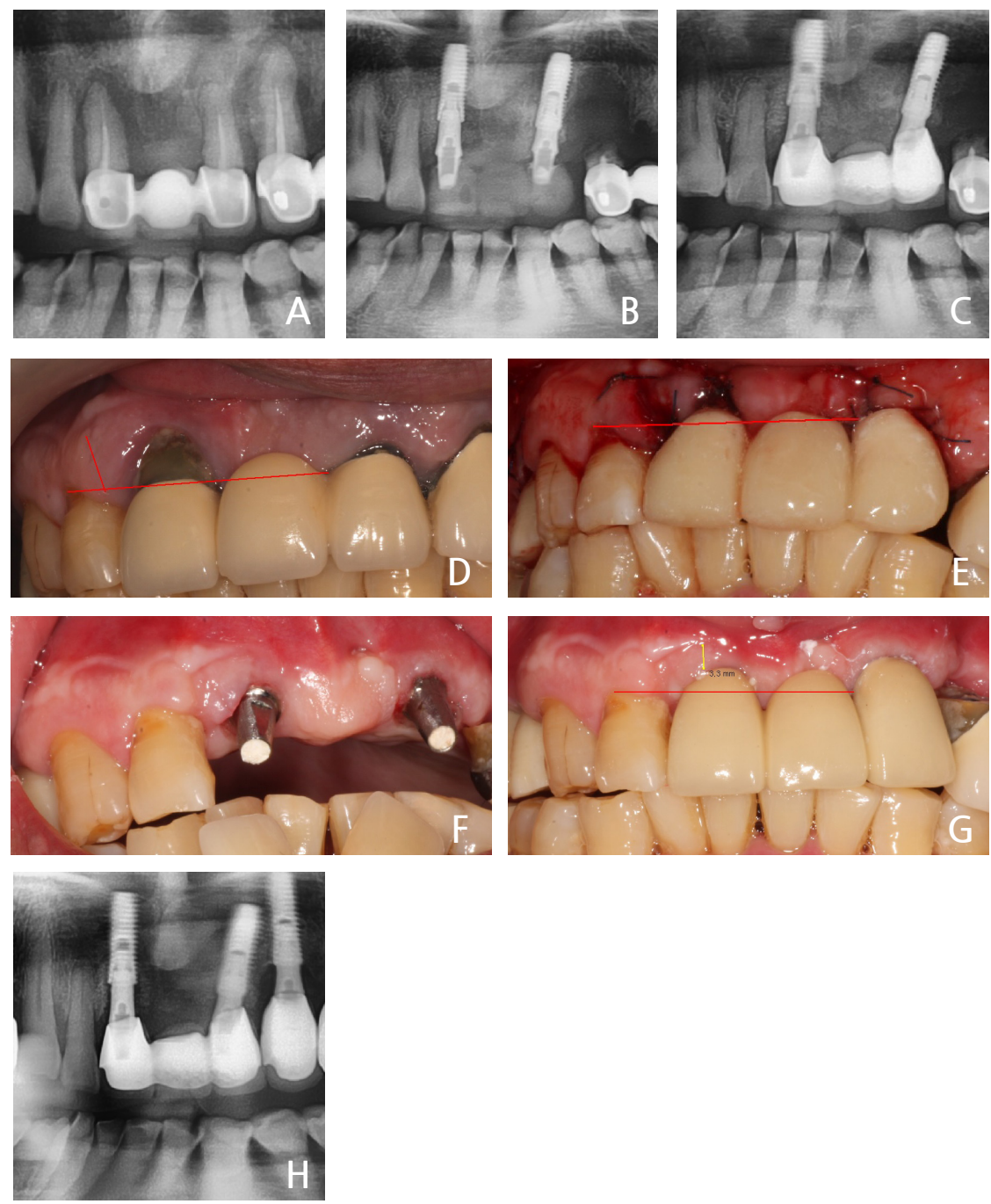

Fig. 7. (A) Bone resorption on the maxilla anterior area, (B) Immediate implant placement with bone graft, (C) Final loading after 2 weeks, (D) Preoperative gingiva recession on the right maxillary incisor, (E) Postoperative immediate provisional crown (\#12 distal proximal gingiva rotational flap for the attached gingiva), (F) Gingival healing after two weeks, (G) Maintained gingiva tissue after 3 weeks postoperatively (final restoration, red arrow: about $3 \mathrm{~mm}$ of attached gingiva), (H) Panoramic view of the 3-years follow-up. 


\section{Case 8}

A 50-year old man visited our clinic because of a fractured right maxillary 1st molar. Clinical and radiographic evaluations showed a decayed residual sinus-invading root (Fig. 8A). After extraction of the residual root, the immediate implant placement was performed with sinus elevation (Fig. 8B). During extraction, the crest area of the buccal bone plate was fractured. The fixture was placed slightly in the palatal area. Primary stability was attained from the inferior sinus wall and intact septal bone. After 3 months, loading was initiated, and a slight recession of the buccal area was observed (Fig. 8C). In radiographic view of 3-years follow-up, it was observed that the normal condition was maintained (Fig. 8D).
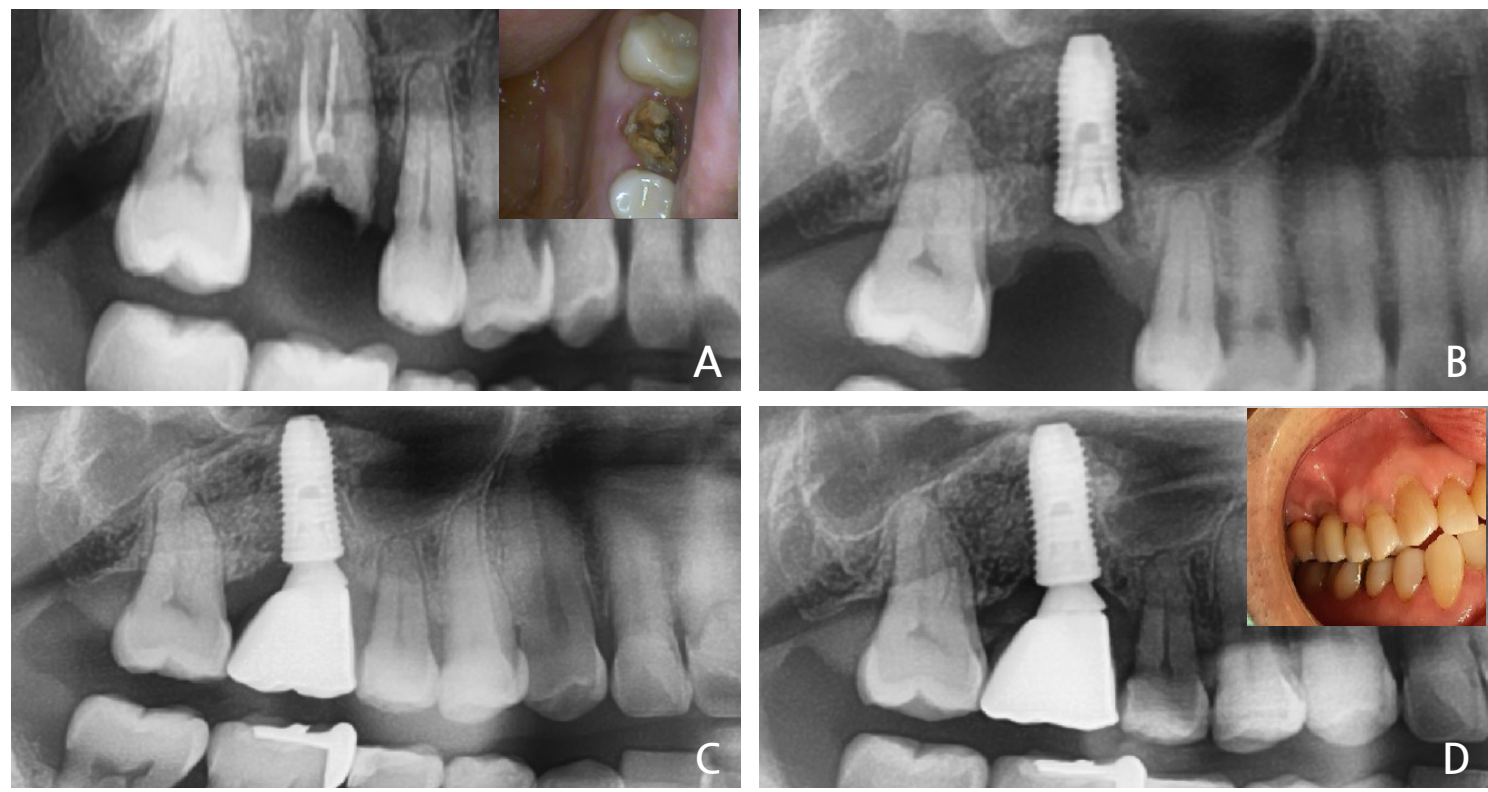

Fig. 8. (A) Decayed residual root of the right maxillary 1st molar, (B) Immediate implant placement with sinus lift, (C) Loading after 3 months, (D) Panoramic view at the 3-years follow-up.

\section{Case summary}

\begin{tabular}{lccccc} 
& Tooth number & Fixture size & Primary stability & Loading & follow up \\
Case 1 & $\# 27$ & $5.0 * 12$ & Inferior wall of the sinus \& intact interseptal bone & 2 months & 3 years \\
Case 2 & $\# 16,17$ & $5.0 * 12$ & Inferior wall of the sinus \& intact interseptal bone & 2 months & 3 years \\
Case 3 & $\# 46$ & $5.0 * 12$ & Apical intact bone & 1 months & 3 years \\
Case 4 & $\# 45$ & $4.5 * 10$ & Linguo-apical intact bone & 1 months & 3 years \\
Case 5 & $\# 35,37$ & $4.0 * 10,5.0 * 8$ & Mesio-lingual intact bone & 1 months & 3 years \\
Case 6 & $\# 47$ & $4.5 * 10$ & Buccal intact bone & 2 months & 2 years \\
Case 7 & $\# 11,22$ & $4.0 * 12$ & Apical intact bone & 2 months & 3 years \\
Case 8 & $\# 16$ & $5.0 * 12$ & Inferior wall of the sinus \& intact interseptal bone & 3 months & 3 years \\
\hline
\end{tabular}




\section{Discussion}

Immediate implant placement has become widely accepted despite the controversial beginning. The primary advantages of immediate implant placement are a reduction in time and surgical episodes and preservation of the bone and gingival tissues. ${ }^{17}$ One of the factors involved in the success of osseous integration and the long-term success of implants is the implant primary stability; it is defined as the biometric stability of the implant immediately after its placement within the bone. Primary stability is related to the mechanical engagement of the implant with the surrounding bone after implant insertion. Secondary stability depends on bone formation and remodeling at the implant-bone interface, and is influenced by the implant surface and wound-healing time. ${ }^{18}$ In this study, primary stability was the most important factor for the loading of an implant with a prosthesis and its success. In clinical practice, the major indication for this method is the ability to attain primary stability. To gain a maximal degree of stability, implants must be placed at $3.0-5.0 \mathrm{~mm}$ to the intact residual bone beyond the apex. ${ }^{19}$ In the case of multi-rooted teeth, the implant can be placed in the inter-septal bone for stability. Also, the cortical bone of the inferior wall of the sinus can be a factor in primary stability. In the mandibular molar area, there is sufficient bone for primary stability in the buccal area of the inferior alveolar nerve. Immediate prosthetic loading is a viable strategy for reducing the time of treatment. This method is certainly an esthetic and functional benefit to the patient, who can avoid wearing uncomfortable removable dentures during the healing period. However, there is a risk of immediate loading of dental implants. The presence of micro-movements can affect bone healing and osseointegration, leading to implant mobilization and failure. ${ }^{19}$ The placement of the immediate implant and immediate loading protocols present predictable solutions today, characterized by high rates of survival and success. However, there is no doubt that these methods are more challenging for the clinician when compared to more conventional protocols. ${ }^{20}$

The placement of implants in extraction sockets can be difficult. In addition, it can be difficult to secure primary stability and complete debridement of infected tissue. In case 6 , the fixture was planted buccally to obtain sufficient initial stability. Thus, the resin hole was positioned on the lingual side of the crown.

In conclusion, the outcomes in this study were satisfactory and showed that immediate implant placement could be an alternative to delayed implant placement. Further prospective clinical studies are warranted to validate the results of this study. 


\section{IV . Conclusion}

Immediate implant placement demonstrated acceptable clinical and radiographic outcomes over 3 years in all 8 patients with 11 implants. In all cases, all implants exhibited primary stability. There were no signs of inflammation or infection, and none of the patients had subsequent complaints. All implants were osseointegrated, and no complications were observed.

\section{References}

1. Schulte W, Kleineikenscheidt H, Lindner K, Schareyka R. The Tübingen immediate implant in clinical studies. Dtsch Zahnarztl Z 1978;33:348-59.

2. Lang NP, Pun L, Lau KY, Li KY, Wong MCM. A systematic review on survival and success rates of implants placed immediately into fresh extraction sockets after at least 1 year. Clin Oral Implants Res 2012;23:39-66.

3. Chen ST, Wilson TG, Jr., Hämmerle CH. Immediate or early placement of implants following tooth extraction: review of biologic basis, clinical procedures, and outcomes. Int J Oral Maxillofac Implants 2004;19:12-25.

4. Gelb DA. Immediate implant surgery: three-year retrospective evaluation of 50 consecutive cases. Int J Oral Maxillofac Implants 1993;8:388-99.

5. Schwartz-Arad D, Gulayev N, Chaushu G. Immediate versus non-immediate implantation for fullarch fixed reconstruction following extraction of all residual teeth: a retrospective comparative study. J Periodontol 2000;71:923-8.

6. Becker W, Dahlin C, Becker BE, Lekholm U, van Steenberghe D, Higuchi K, et al. The use of e-PTFE barrier membranes for bone promotion around titanium implants placed into extraction sockets: a prospective multicenter study. Int J Oral Maxillofac Implants 1994;9:31-40.

7. Huys LW. Replacement therapy and the immediate post-extraction dental implant. Implant Dent 2001;10:93-102.

8. Polizzi G, Grunder U, Goené R, Hatano N, Henry P, Jackson WJ, et al. Immediate and delayed implant placement into extraction sockets: a 5-year report. Clin Implant Dent Relat Res 2000;2:93-9.

9. Schwartz D, Chaushu G. The ways and wherefores of immediate placement of implants into fresh extraction sites: a literature review. J Periodontol 1997;68:915-23.

10. Siegenthaler DW, Jung RE, Holderegger C, Roos M, Hämmerle CH. Replacement of teeth exhibiting periapical pathology by immediate implants: a prospective, controlled clinical trial. Clin Oral Implants Res 2007; 18:727-37.

11. Brazily I. Immediate implants: their current status. Int J Prosthodont 1993;6:169-75.

12. Novaes AB Jr, Marcaccini AM, Souza SLS, Taba M Jr, Grisi MFM. Immediate placement of implants into periodontally infected sites in dogs: a histomorphometric study of bone-implant contact. Int J Oral Maxillofac Implants 2003;18:391-8.

13. Novaes AB Jr, Vidigal GM Jr, Novaes AB, Grisi MFM, Polloni S, Rosa A. Immediate implants placed into infected sites: A histomorphometric study in dogs. Int J Oral Maxillofac Implants $1998 ; 13: 422-7$. 
14. Crespi R, Cappare P, Gherlone E. Fresh-socket implants in periapical infected sites in humans. J Periodontol 2010;81:378-83.

15. Villa R, Rangert B. Early loading of interforaminal implants immediately installed after extraction of teeth presenting endodontic and periodontal lesions. Clin Implant Dent Relat Res 2005; 7:S28-35.

16. Coatoam GW, Mariotti A, 2000. Immediate placement of anatomically shaped dental implants. J Oral Implantol 26:170-6.

17. Bhola M, Neely AL, Kolhatkar S. Immediate implant placement: clinical decisions, advantages, and disadvantages. J Prosthodont 2008; 17:576-81.

18. Quesada-García MP, Prados-Sánchez E, Olmedo-Gaya MV, Muñoz-Soto E, González-Rodríguez MP, Vallecillo-Capilla M. Measurement of dental implant stability by resonance frequency analysis: a review of the literature. Med Oral Patol Oral Cir Bucal 2009;14:e538-46.

19. Stanley M, Braga FC, Jordao BM. Immediate loading of single implants in the anterior maxilla: a 1-year prospective clinical study on 34 patients. Int J Dent 2017;8346496.

20. Chrcanovic B.R. Albrektsson T. Wennerberg A. Dental implants inserted in fresh extraction sockets versus healed sites: a systematic review and meta-analysis. J Dent 2015;43:16-41. 